\title{
Lunar occultations for December. Occultations of Planets and fixed Stars by the Moon, in December 1831. Computed for Greenwich and circulated by the Astronomical Society
}

\author{
Thomas Henderson Esq.
}

To cite this article: Thomas Henderson Esq. (1831) Lunar occultations for December. Occultations of Planets and fixed Stars by the Moon, in December 1831. Computed for Greenwich and circulated by the Astronomical Society , Philosophical Magazine Series 2, 10:60, 470-470, DOI: $10.1080 / 14786443108674313$

To link to this article: http://dx.doi.org/10.1080/14786443108674313

曲 Published online: 14 Jul 2009.

Submit your article to this journal $₫$

Џ Article views: 2

Q View related articles $\sqsubset$ 
LUNAR OCCULTATIONS FOR DECEMBER.

Occultations of Planets and fixed Stars by the Moon, in December 1831. Computed for Greenwich, by Thomas Henderson, Esq.; and circulated by the Astronomical Society.

\begin{tabular}{|c|c|c|c|c|c|c|c|c|c|c|c|}
\hline \multirow[b]{3}{*}{1831.} & \multirow{3}{*}{$\begin{array}{l}\text { Stars' } \\
\text { Names. }\end{array}$} & \multirow{3}{*}{ 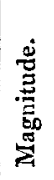 } & \multirow{3}{*}{ 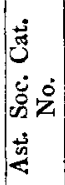 } & \multicolumn{4}{|c|}{ Immersions. } & \multicolumn{4}{|c|}{ Emersions. } \\
\hline & & & & \multirow[b]{2}{*}{$\begin{array}{c}\text { Sidereal } \\
\text { time. }\end{array}$} & \multirow[b]{2}{*}{$\begin{array}{l}\text { Mean } \\
\text { solar time }\end{array}$} & \multicolumn{2}{|c|}{ Angle from } & \multirow[b]{2}{*}{$\begin{array}{c}\text { Sidereal } \\
\text { time. }\end{array}$} & \multirow[b]{2}{*}{$\begin{array}{l}\text { Mean } \\
\text { solartime }\end{array}$} & \multicolumn{2}{|c|}{ Angle from } \\
\hline & & & & & & 劳远 & 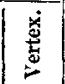 & & & 至 & 总 \\
\hline \multirow{16}{*}{ Dec. } & & & & h $\mathrm{m}$ & h $\mathrm{m}$ & & & $\mathrm{h} \mathbf{m}$ & h $\mathrm{m}$ & & \\
\hline & ${ }_{a}$ Capricor.* & 6 & 2408 & 2130 & 423 & 174 & 185 & $\begin{array}{|ll|}21 & 57\end{array} \mid$ & 451 & 214 & 229 \\
\hline & 42 Aquarii & 6 & 2653 & 26 & 851 & 95 & 128 & Under & horizon. & $\ldots$ & $\ldots$ \\
\hline & $\xi^{2}$ Cet & 5 & 255 & 2358 & 623 & 103 & 76 & $\begin{array}{ll}1 & 6\end{array}$ & 731 & 307 & 291 \\
\hline & $\mu$ Cet & 4 & 293 & 856 & 1520 & 125 & 164 & Under & horizon. & $\ldots$ & $\ldots$ \\
\hline & $f$ Taur & $5 \cdot 6$ & 379 & 250 & 911 & 53 & 46 & 328 & 949 & 349 & 350 \\
\hline & Tau & $3 \cdot 4$ & 478 & $21 \quad 19$ & 337 & 99 & 61 & $22 \quad 10$ & 4 & 293 & 253 \\
\hline & $\mathrm{T}$ & 6 & 508 & $\begin{array}{ll}0 & 59\end{array}$ & 716 & 128 & 90 & $\begin{array}{ll}1 & 59\end{array}$ & 817 & $268 \mid$ & 238 \\
\hline & $\mathrm{Ta}$ & 5 & 510 & 118 & 735 & 18 & 343 & \multirow{2}{*}{\multicolumn{4}{|c|}{$\begin{array}{l}\text { Dalmost touching Star- } \\
\text { Occulted to places further } \\
\text { North. }\end{array}$}} \\
\hline & & & & & & & & & & & \\
\hline & (99) Tami & $5 \cdot 6$ & 516 & 155 & 812 & 61 & 29 & 242 & 859 & 333 & 310 \\
\hline & Aldeb & 1 & 528 & 446 & 112 & 48 & $5 \hat{3}$ & 525 & 1142 & 340 & 355 \\
\hline & 119 Tauri & $5 \cdot 6$ & 663 & 058 & 711 & 120 & 79 & 155 & 89 & 266 & 227 \\
\hline & 1207 & 6 & 667 & 127 & 740 & 96 & 56 & $\begin{array}{ll}2 & 29\end{array}$ & 842 & 290 & 254 \\
\hline & $g$ Ger & 6 & 951 & 249 & 855 & 35 & 354 & 323 & 928 & 325 & 284 \\
\hline & Regulus.. & 1 & 1209 & 1344 & 1939 & 68 & 105 & \begin{tabular}{ll|}
14 & 45
\end{tabular} \mid & 2040 & 250 & 290 \\
\hline
\end{tabular}

* Double Star.

\section{METEOROLOGICAL OBSERVATIONS FOR OCTOBER 1831.}

Gosport:-Numerical Results for the Month.

Barom. Max. 30-428. Oct. 17. Wind N.-Min.29.231. Oct. 1. Wind S. Range of the mercury $1 \cdot 197$.

Mean barometrical pressure for the month $29 \cdot 904$

Spaces described by the rising and falling of the mercury............ 4.865

Greatest variation in 24 hours 0.594 . - Number of changes 16 .

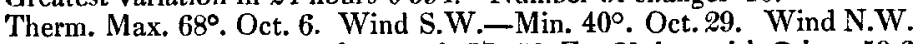

Range $28^{\circ}$.-Mean temp. of exter. air $57^{\circ} \cdot 58$. For 31 days with $\odot$ in $\approx 59 \cdot 68$

Max. var. in 24 hours $19^{\circ} \cdot 00$. - Mean temp. of spring water at 8 A.M. 54.30

\section{De Luc's Whalebone Hygrometer.}

Greatest humidity of the atmosphere, in the evening of the 12th.... $97^{\circ}$

Greatest dryness of the atmosphere, in the afternoon of the 5th...... 61.0

Range of the index .............................................. 36.0

Mean at 2 P.M. $72 \circ 6$. Mean at 8 A.M. $78^{\circ} 1 .-$ Mean at 8 P.M. $\quad 82 \cdot 0$

- of three observations each day at 8,2 , and 8 o'clock ......... $77 \cdot 6$

Evaporation for the month $1 \cdot 60$ inch.

Rain in the pluviameter near the ground 4.835 inches.

Prevailing wind, South-west.

Summary of the Weather.

A clear sky, 2 ; fine, with various moditications of clouds, 13 ; an overcast sky without rain, 8 ; foggy, $\frac{1}{2}$; rain, $7 \frac{1}{2}$.- Total 31 days.

Clouds. 